OPEN ACCESS

Edited by:

Jose Ramon Pineda,

University of the Basque

Country, Spain

Reviewed by:

Patricia Garcia Gallastegi,

University of the Basque

Country, Spain

Hongjuan Cui,

Southwest University, China

*Correspondence: Zhi-Qiang Li

lizhiqiang@whu.edu.cn

†These authors have contributed equally to this work

Specialty section: This article was submitted to Neuro-Oncology and Neurosurgical

Oncology,

a section of the journal Frontiers in Neurology

Received: 04 July 2020 Accepted: 31 August 2020 Published: 07 October 2020

Citation:

Yang $C$, Wen $H$-B, Zhao $Y$ - $H$, Huang $W-H$, Wang Z-F and Li Z-Q

(2020) Systemic Inflammatory Indicators as Prognosticators in

Glioblastoma Patients: A

Comprehensive Meta-Analysis.

Front. Neurol. 11:580101.

doi: 10.3389/fneur.2020.580101

\title{
Systemic Inflammatory Indicators as Prognosticators in Glioblastoma Patients: A Comprehensive Meta-Analysis
}

\section{Chao Yang ${ }^{1+}$, Hong-Bin Wen ${ }^{2+}$, Yu-Hang Zhao ${ }^{1}$, Wen-Hong Huang ${ }^{1}$, Ze-Fen Wang ${ }^{3}$ and Zhi-Qiang Li ${ }^{\text {1* }}$}

${ }^{1}$ Department of Neurosurgery, Zhongnan Hospital of Wuhan University, Wuhan, China, ${ }^{2}$ Department of Neurology, Xiangyang Central Hospital, Xiangyang, China, ${ }^{3}$ Department of Physiology, School of Basic Medical Science, Wuhan University, Wuhan, China

Background: Inflammation plays an important role in tumorigenesis. Previous studies have reported the prognostic value of several peripheral inflammatory markers in glioma patients, including the neutrophil-to-lymphocyte ratio (NLR). However, it still remains unclear whether inflammatory markers can independently predict the prognosis of glioblastoma (GBM) patients. The present study aims to explore the prognostic value of systemic inflammatory markers, including neutrophils, lymphocytes, platelets, the NLR, and the platelet-to-lymphocyte ratio (PLR), in patients with GBM.

Methods: A comprehensive systemic search and review was performed using the PubMed, EMBASE, and Cochrane Library databases to identify all the relevant literature (published before June 30, 2020) that evaluated the association between any of these inflammatory markers and survival in GBM.

Results: There were 2 (634 patients), 3 (723 patients), 2 (237 patients), 8 (1,225 patients), and 3 (505 patients) studies examining the correlation of survival with neutrophils, lymphocytes, platelets, the NLR, and the PLR, respectively. An elevated NLR and elevated neutrophil and platelet counts were associated with worse overall survival (OS) in GBM patients (NLR: hazard ratio $[H R]=1.63,95 \%$ confidence interval [Cl]: 1.23-2.15, $p=0.0007$; neutrophil count: $\mathrm{HR}=1.46,95 \% \mathrm{Cl}: 1.16-1.83, p=0.001$; platelet count: $\mathrm{HR}=1.58,95 \% \mathrm{Cl}: 1.42-1.77, p<0.00001)$. However, there was no significant association between the PLR or the absolute lymphocyte count and OS in GBM patients.

Conclusion: The NLR and the absolute neutrophil and platelet counts may be valuable and convenient peripheral inflammatory markers to evaluate the prognosis of GBM patients. Further prospective studies are needed to verify its reliability.

Keywords: neutrophils, lymphocytes, platelets, NLR, PLR, glioblastoma, overall survival 


\section{INTRODUCTION}

Gliomas are the most common primary malignant brain tumors in adults. Glioblastoma (GBM) is the most lethal type of glioma and has a highly aggressive clinical course with a median survival time of $\sim 14$ months (1). Genome-wide molecular profiling studies have revealed many classic genetic alterations in different types of gliomas. Molecular biomarkers such as isocitrate dehydrogenase $1(\mathrm{IDH} 1)$ and $\mathrm{O}^{6}$-methylguanine-DNA methyltransferase (MGMT) play an important role in evaluating the prognosis of glioma patients $(2,3)$. In the revised 2016 WHO Classification of Tumors of the central nervous system, molecular phenotypes were added to the classifications of gliomas for the first time, increasing our understanding of glioma and its prognosis (4). Although IDH1 mutation and MGMT promoter methylation were already thought to be important molecular markers, disadvantages in testing techniques have impeded their widespread application. Therefore, finding additional markers for predicting the outcomes of patients with glioma that are easy to test is urgent and necessary. Neutrophil, lymphocyte, and platelet counts, as well as the neutrophil-to-lymphocyte ratio (NLR) and the platelet-to-lymphocyte ratio (PLR), can be easily obtained from routine blood examination; however, disputes on their prognostic value in GBM still exist.

Over the last decade, it has become clear that inflammation plays an important role in tumorigenesis. Long-term chronic inflammation promotes tumor initiation and progression (5), and inflammation can increase the proliferation and survival of tumor cells. The blood supply of tumors is also improved by inflammation (6). Neutrophils, lymphocytes, and platelets are classic inflammatory cells, and their levels in the peripheral blood are tightly associated with the extent of inflammation. As with other cancer patients, most glioma patients experience strong neutrophilia and lymphopenia because of overproduction of Granulocyte Colony Stimulating Factor (G-CSF) by tumor cells. G-CSF diverts bone-marrow hematopoiesis away from the lymphocyte lineage toward the granulocyte lineage (7). A review by Donskov reported that elevated neutrophils both in tumor around and in peripheral blood, and a high NLR associated with poor clinical outcomes in several human cancers, such as renal cell carcinoma, hepatocellular carcinoma, colorectal cancer, and so on (8). Especially, the significance of NLR has been largely studied in colorectal cancer. An elevated NLR was observed to independently predict poorer outcomes in advanced colorectal cancer patients with oxaliplatin-based chemotherapy (9) and with liver metastasis (10). Some reports have shown that a higher level of neutrophils predicted a worse outcome in GBM patients $(11,12)$. However, Lopes et al. reported that the neutrophil count does not correlate with prognosis in GBM patients (13). Most studies did not find prognostic value for the lymphocyte count in GBM, possibly because of the various subgroups of lymphocytes. However, one study reported that treatment-related lymphopenia after surgery could independently predict the prognosis of GBM patients (14). A recent review showed that thrombocytosis at the time of diagnosis is associated with shorter survival in many solid tumors, including malignant glioma (15), but other studies have reported that the platelet level cannot predict prognosis in GBM $(13,16)$. Previous studies have explored the prognostic value of the NLR and PLR in many cancers, including gastric cancer (17), breast cancer (18), primary liver cancer (19), advanced esophageal cancer (20), prostate cancer (21), and renal cell carcinoma (22). Compared to traditional molecular prognostic markers, such as IDH1 mutation and MGMT promoter methylation status (23), these hematological inflammatory markers can conveniently evaluate the prognosis of GBM patients in order to guide clinicians' therapeutic decisions and patient management.

There have been two meta-analysises $(24,25)$ evaluating the prognostic role of the NLR in patients with glioma recently. However, one of them only analyzed NLR and both of them overlooked the influence of the type of variable on the final results. To our knowledge, this is the first meta-analysis to systematically assess more inflammatory markers and offer a better understanding of the prognostic value of the neutrophil, lymphocyte, and platelet counts in GBM patients.

\section{MATERIALS AND METHODS}

The Preferred Reporting Items for Systematic Reviews and MetaAnalysis (PRISMA) guidelines were used to perform this metaanalysis (26). A completed PRISMA 2009 Checklist was shown in Supplementary File 1.

\section{Search Strategy}

We performed a systematic review to identify articles published before June 30, 2020 from the PubMed, EMBASE, and Cochrane Library databases assessing the association between any one of the five inflammatory markers and prognosis in GBM patients. We performed five searches based on different inflammatory markers. Our five searches included the Mesh Terms: (1) "NLR" and "Glioma;" (2) "PLR" and "Glioma;" (3) "Neutrophils" and "Glioma" and "Prognosis;" (4) "Lymphocytes" and "Glioma" and "Prognosis;" and (5) "Blood Platelets" and "Glioma" and "Prognosis." The relevant free terms are listed in the Supplementary File 2. Reviews and the references of included studies were also checked to avoid omission of relevant publications.

\section{Eligibility Criteria}

We included studies only if all the following conditions were met: (1) included patients diagnosed with GBM; (2) either preoperative or postoperative inflammatory indices (neutrophils, lymphocytes, platelets, NLR, and PLR) were evaluated using multivariate analysis; and (3) hazard ratios (HRs) and 95\% confidence intervals (CIs) for overall survival (OS) were provided directly from the multivariate analysis. Studies were excluded if they met the following criteria: (1) not in humans, or (2) not published in English; (3) case reports, letters, conference abstracts and non-clinical studies; (4) duplicated publications.

\section{Study Selection and Data Extraction}

Two reviewers (C.Y. and Y.H.Z.) independently selected relevant studies that met our inclusion criteria and extracted the relevant information, with any disagreements resolved by discussion to 
achieve a consensus. Publications were read for extraction of original data. The main data extracted were HRs with 95\% CIs. Characteristics of the studies were also extracted, including first author, publication year, country, number of patients (female/male ratio), age, glioma grade, sampling time (before or after surgery), types of variables, and the cut-off value of inflammatory markers.

\section{Quality Evaluation}

The Newcastle-Ottawa Scale was used to assess the quality and risk of bias of the included studies. Two authors independently conducted the assessment of the included studies based on three main aspects, including study selection ( $0-4$ points), comparability ( $0-2$ points), and study outcomes ( $0-3$ points). The maximum score is nine points and high scores represent high quality and low risk of bias (Table 1).

\section{Statistical Analysis}

Time-to-event data (OS) was analyzed by the HR. Forest plots were drawn to obtain the pooled $\mathrm{HR}$, which was considered

TABLE 1 | Quality evaluation of included studies using the Newcastle Ottawa Scale for cohort studies.

\begin{tabular}{|c|c|c|c|c|c|c|c|c|c|c|c|}
\hline \multirow[t]{2}{*}{ Markers } & \multirow[t]{2}{*}{ Author } & \multirow[t]{2}{*}{ Year } & \multicolumn{4}{|c|}{ Selections } & \multirow{2}{*}{$\begin{array}{c}\text { Comparability } \\
\text { E }\end{array}$} & \multicolumn{4}{|c|}{ Outcome Score } \\
\hline & & & A & B & C & D & & $\mathbf{F}$ & G & $\mathbf{H}$ & \\
\hline \multirow[t]{2}{*}{ Neutrophil } & Mason & 2017 & 1 & 1 & 1 & 0 & 2 & 1 & 1 & 1 & 8 \\
\hline & Bertaut & 2016 & 1 & 1 & 1 & 0 & 1 & 1 & 1 & 1 & 7 \\
\hline \multirow[t]{3}{*}{ Lymphocyte } & Lopes & 2018 & 1 & 1 & 1 & 0 & 1 & 1 & 1 & 1 & 7 \\
\hline & Mason & 2017 & 1 & 1 & 1 & 0 & 2 & 1 & 1 & 1 & 8 \\
\hline & Han & 2015 & 1 & 1 & 1 & 0 & 2 & 1 & 1 & 1 & 8 \\
\hline \multirow[t]{2}{*}{ Platelet } & Zhou & 2016 & 1 & 1 & 1 & 0 & 1 & 1 & 1 & 1 & 7 \\
\hline & Brockmann & 2007 & 1 & 1 & 1 & 0 & 1 & 1 & 1 & 1 & 7 \\
\hline \multirow[t]{8}{*}{ NLR } & Mason & 2017 & 1 & 1 & 1 & 0 & 2 & 1 & 1 & 1 & 8 \\
\hline & Wang PF & 2017 & 1 & 1 & 1 & 0 & 2 & 1 & 1 & 1 & 8 \\
\hline & Han & 2015 & 1 & 1 & 1 & 0 & 2 & 1 & 1 & 1 & 8 \\
\hline & Bambury & 2013 & 1 & 1 & 1 & 0 & 1 & 1 & 1 & 1 & 7 \\
\hline & Kaya & 2017 & 1 & 1 & 1 & 0 & 1 & 1 & 1 & 1 & 7 \\
\hline & Weng & 2018 & 1 & 1 & 1 & 0 & 1 & 1 & 1 & 1 & 7 \\
\hline & Brenner & 2019 & 1 & 1 & 1 & 0 & 2 & 1 & 1 & 1 & 8 \\
\hline & Zhang & 2019 & 1 & 1 & 1 & 0 & 1 & 1 & 1 & 1 & 7 \\
\hline \multirow[t]{3}{*}{ PLR } & Wang & 2017 & 1 & 1 & 1 & 0 & 2 & 1 & 1 & 1 & 8 \\
\hline & Han & 2015 & 1 & 1 & 1 & 0 & 2 & 1 & 1 & 1 & 8 \\
\hline & Hao & 2019 & 1 & 1 & 1 & 0 & 2 & 1 & 1 & 1 & 8 \\
\hline
\end{tabular}

A: Representativeness of the exposed cohort: Highly representative or partially representative (one point); no description (no point), B: Selection of the non-exposed cohort: Patients drawn from the same community as the exposed cohort (one point); patients drawn from a different source or no description (no point), C: Ascertainment of exposure: Information obtained from secure record or structured interview (one point); written self-report or no description (no point), D: Demonstration that outcome of interest was not present at start of study: yes (one point), no (no point), E: Comparability of cohorts based on the design or analysis: Study controls for important factor, such as age, ECOG, molecular markers, extent of resection, (two points: All factors were included, one point: Part of them were included), F: Assessment of outcome: Independent blind assessment or record linkage (one point); self-report or no description (no point), G: Follow-up long enough for outcomes to occur: Yes (one point); no (no point), H: Adequacy of follow-up of cohorts: Complete follow up-all subjects accounted for or subjects lost to follow up unlikely to introduce bias (one point); no statement (no point). statistically significant if the 95\% CI did not overlap 1 and the $p<0.05$. Subgroup analysis was performed based on sampling time. The heterogeneity among studies was also evaluated, with $P<0.10$ or $I^{2}>50 \%$ indicating significant heterogeneity (27), and random- or fixed-effects models were adopted when the heterogeneity was or was not significant, respectively. The sources of heterogeneity were evaluated by subgroup analysis. Sensitivity analysis was performed by excluding a single study at a time to examine the stability of the results. Publication bias was evaluated by funnel plot. Statistical difference was defined as $P<0.05$. All the statistical processes were performed using RevMan Version 5.3 (Review Manager, Copenhagen: The Nordic Cochrane Center, The Cochrane Collaboration, 2014).

\section{RESULTS}

\section{Literature Search}

There were 230, 1573, 728, 203, and 70 studies initially identified examining the absolute neutrophil, lymphocyte, and platelet counts and the NLR and PLR, respectively. We removed duplicated studies, and, after full-text evaluation, conference abstract, and articles lacking sufficient data were excluded. One study (13) treated these five inflammatory markers as continuous variables and was excluded in the last quantitative synthesis for these markers, except the lymphocyte count, because of differences in variable type. Likewise, one study (14) treated the lymphocyte count as a dichotomous variable, which was different from the remaining studies, and was therefore excluded in the last quantitative synthesis. Finally, there were $2(11,12), 3(11,14,28)$, $2(16,29), 8(11,30-36)$, and $3(30,31,37)$ included studies examining the neutrophil, lymphocyte, and platelet counts and the NLR and PLR, respectively. All the incorporated studies focused on glioblastoma patients performing the multivariate analysis. The study selection process based on the PRISMA statement (Supplementary File 1) is shown in Figure 1. The main characteristics of the incorporated studies are presented in Table 2.

\section{Association Between the OS and Inflammatory Markers}

We performed five analyses based on the different inflammatory markers.

\section{Neutrophil Count}

Two studies examining 634 patients were included in the analysis. One study (11) from Canada was published in 2017, another from France (12) was published in 2016. The cut-off value are $8,6(10 \mathrm{e} 9 / \mathrm{L})$, respectively. Both of them analyzed the neutrophil count as category variable. The NOS scores are 8, 7 points, respectively. Both studies sampled the neutrophil count postoperatively, and an elevated neutrophil count predicted a poor prognosis in $\mathrm{GBM}$ patients $(\mathrm{HR}=1.46,95 \% \mathrm{CI}$ : $1.16-1.83$, $p=0.001, I^{2}=0 \%$, fixed-effect model) (Figure 2A). We did not perform sensitivity analysis because there were only two studies. 


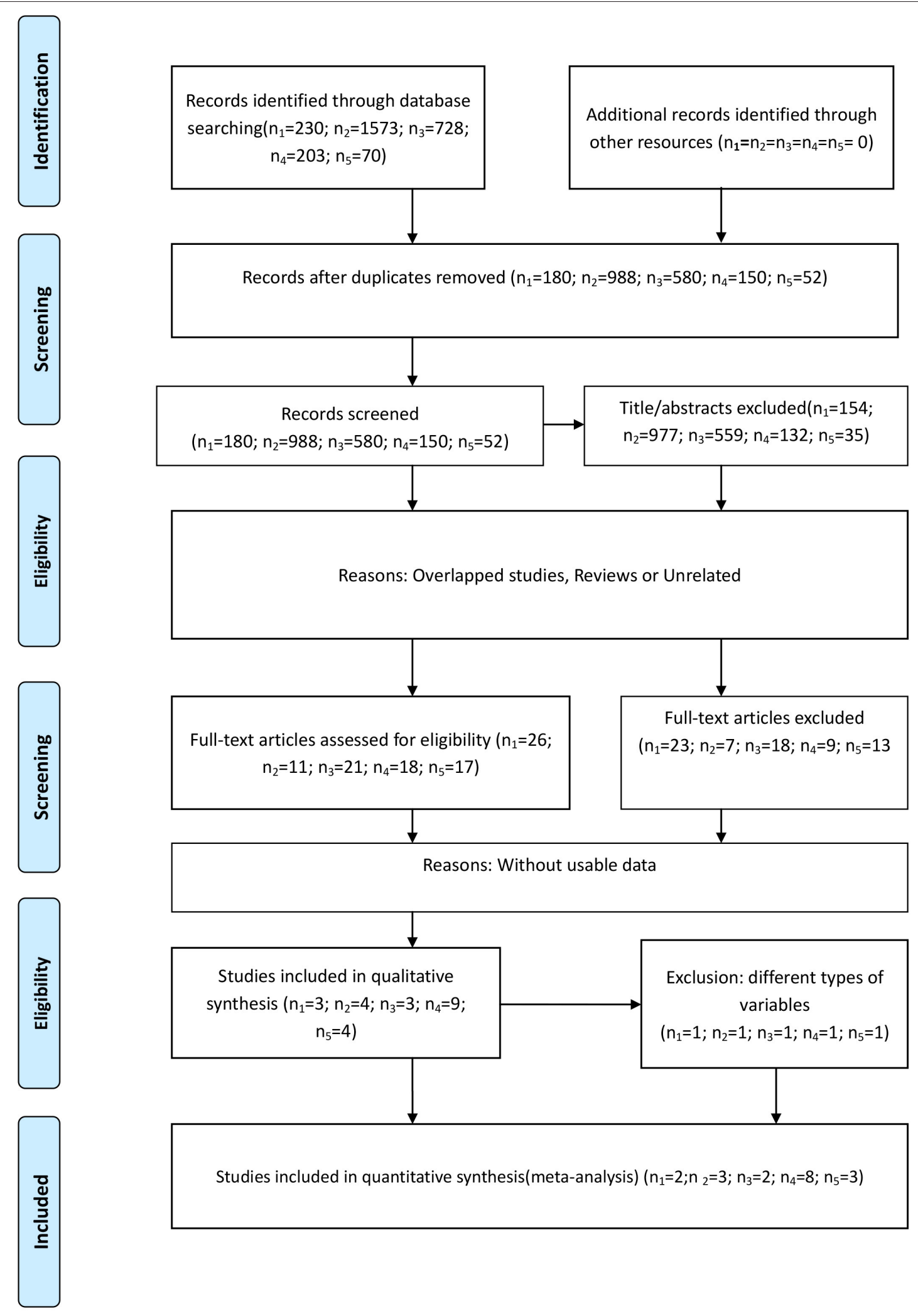

FIGURE 1 | Flow diagram of the study selection process. $\left(n_{1}-n_{5}\right.$ represent the numbers of incorporated literature of neutrophils, lymphocytes, platelets, NLR, and $\mathrm{PLR}$, respectively). 
TABLE 2 | Characteristics of the included studies.

\begin{tabular}{|c|c|c|c|c|c|c|c|c|c|}
\hline Markers & Author & Year & Country & $\mathbf{N}(\mathrm{F} / \mathrm{M})$ & Age & Grade & $\begin{array}{l}\text { Sampling } \\
\text { time }\end{array}$ & Cut-off value & $\begin{array}{l}\text { Multivariate } \\
\text { HR }\end{array}$ \\
\hline \multirow[t]{2}{*}{ Neutrophils ( $\left.{ }^{\star} 10^{9} / \mathrm{L}\right)$} & Mason & 2017 & Canada & 369 (131/238) & Median 55 & IV & Postoperative & 8 & Yes \\
\hline & Bertaut & 2016 & France & $265(106 / 159)$ & $\begin{array}{l}\text { Mean 59.8; } \\
\text { Median 61 }\end{array}$ & IV & Postoperative & $6,000 / \mathrm{mm}^{3}$ & Yes \\
\hline \multirow[t]{3}{*}{ Lymphocytes (*109/L) } & Lopes & 2018 & Portugal & $140(42 / 98)$ & Mean 62.9 & IV & Preoperative & $\backslash$ & Yes \\
\hline & Mason & 2017 & Canada & $369(131 / 238)$ & Median 55 & IV & Postoperative & 1 & Yes \\
\hline & Han & 2015 & China & $214(94 / 120)$ & Mean 52.3 & IV & Preoperative & 1 & yes \\
\hline \multirow[t]{2}{*}{ Platelets $\left({ }^{\star} 10^{9} / \mathrm{L}\right)$} & Zhou & 2016 & China & $84(34 / 50)$ & Median 53 & IV & Preoperative & 151 & Yes \\
\hline & Brockmann & 2007 & Germany & $153(63 / 90)$ & $\backslash$ & IV & Preoperative & $\backslash$ & yes \\
\hline \multirow[t]{8}{*}{ NLR } & Mason & 2017 & Canada & $369(131 / 238)$ & Median 55 & IV & Postoperative & 7.5 & Yes \\
\hline & Wang PF & 2017 & China & $166(70 / 96)$ & Mean 52.1 & IV & Preoperative & 4 & Yes \\
\hline & Han & 2015 & China & $152(57 / 95)$ & Mean 50.4 & IV & Preoperative & 4 & Yes \\
\hline & Bambury & 2013 & Ireland & $84(19 / 65)$ & Median 58 & IV & Preoperative & 4 & Yes \\
\hline & Kaya & 2017 & Turkey & $90(39 / 51)$ & $\begin{array}{l}\text { Mean 55.7; } \\
\text { Median 58.5 }\end{array}$ & IV & Preoperative & 5 & Yes \\
\hline & Brenner & 2019 & Israel & $89(43 / 46)$ & Median 59 & IV & Preoperative & 4 & Yes \\
\hline & Zhang & 2019 & China & $170(-)$ & $\backslash$ & IV & Preoperative & 7.25 & Yes \\
\hline & Weng & 2018 & China & 105 (52/53) & $\backslash$ & IV & Preoperative & 4 & Yes \\
\hline \multirow[t]{3}{*}{ PLR } & Wang & 2017 & China & $166(70 / 96)$ & Mean 52.1 & IV & Preoperative & 175 & Yes \\
\hline & Han & 2015 & China & $152(57 / 95)$ & Mean 50.4 & IV & Preoperative & 135 & Yes \\
\hline & $\mathrm{HaO}$ & 2019 & China & $187(71 / 116)$ & Mean 55 & IV & preoperative & 228.6 & Yes \\
\hline
\end{tabular}

N (F/M): numbers of patients (female/male), Grade: the pathological grade of glioma, Sampling time: the blood was obtained preoperatively or postoperatively, HR: hazard ratio.

\section{Platelet Count}

Two studies examining 237 patients were examined for this analysis. One from China (16) was published in 2016 and another from Germany (29) was published in 2007. The cut-off value are 151, 400(10e9/L), respectively, and all the NOS scores are 7 points. Both sampled the platelet count preoperatively and evaluated it as a dichotomous variable. Elevated platelets also predicted a bad outcome in patients with GBM ( $\mathrm{HR}=1.58$, 95\% CI: $1.42-1.77, p<0.00001, I^{2}=0 \%$, fixed-effect model) (Figure 2B).

\section{NLR}

We included eight retrospective studies (11, 30-36) published between 2013 and 2019 examining 1,225 patients to evaluate the association between the NLR and prognosis in GBM patients. There are 4 studies from China, 1 from Canada, 1 from Ireland, 1 from Turkey, and 1 from Israel. Five studies used 4 as the cut-off value, while the other three studies used $7.5,7.25$, and 5 as the cut-off value, respectively. The NOS of 4 studies were 8 points and the other 4 studies were 7 points. In all patients, a higher NLR predicted a worse prognosis in GBM patients $(\mathrm{HR}=1.63$, 95\% CI: $1.23-2.15, p=0.0007, I^{2}=65 \%$, random-effect model) (Figure 2C). Seven studies sampled preoperatively, whereas the study by Mason et al. (11) sampled postoperatively, and, in the sensitivity analysis, it was the major source of the heterogeneity. After excluding this study, the heterogeneity was 32\% $(p=0.19)$, and the pooled $\mathrm{HR}$ remained statistically significant $(\mathrm{HR}=1.79$, 95\% CI: 1.42-2.27, $p<0.00001)$.

\section{Lymphocyte Count}

Three studies were included in this analysis, and the pooled HR for OS was 0.89 (95\% CI: 0.77-1.03) (Figure 3A), indicating that the peripheral lymphocyte count did not have prognostic value in patients with GBM. Sensitivity analysis showed that the result was stable. One study from Portugal (13) was published in 2018, one from Canada (11) was published in 2017 and the last one from China (28) was published in 2015. All the incorporated studies analyzed the lymphocyte count as the continuous variable. Two studies sampled preoperatively and one sampled postoperatively. The NOS are $7,8,8$ points, respectively. In further subgroup analysis, the pooled HR of the lymphocyte count for the two studies assessing lymphocyte count before surgery was 0.88 (95\% CI: $0.76-1.02)$.

\section{PLR}

We included three studies examining 505 patients, and, in our meta-analysis, the PLR was not associated with the prognosis of $\mathrm{GBM}$ patients $(\mathrm{HR}=1.41,95 \% \mathrm{CI}: 0.94-2.10, p=0.09)$ (Figure 3B). All the three studies $(30,31,37)$ published between 2015 and 2019 were from China and sampled preoperatively. The cut-off value were $175,135,228.6$, respectively, and the NOS were all 8 points.

\section{Publication Bias}

Due to limitations in the quantity of included studies, we only tested the publication bias in studies examining the NLR. No significant publication bias was found in the present metaanalysis (Figure 4). 


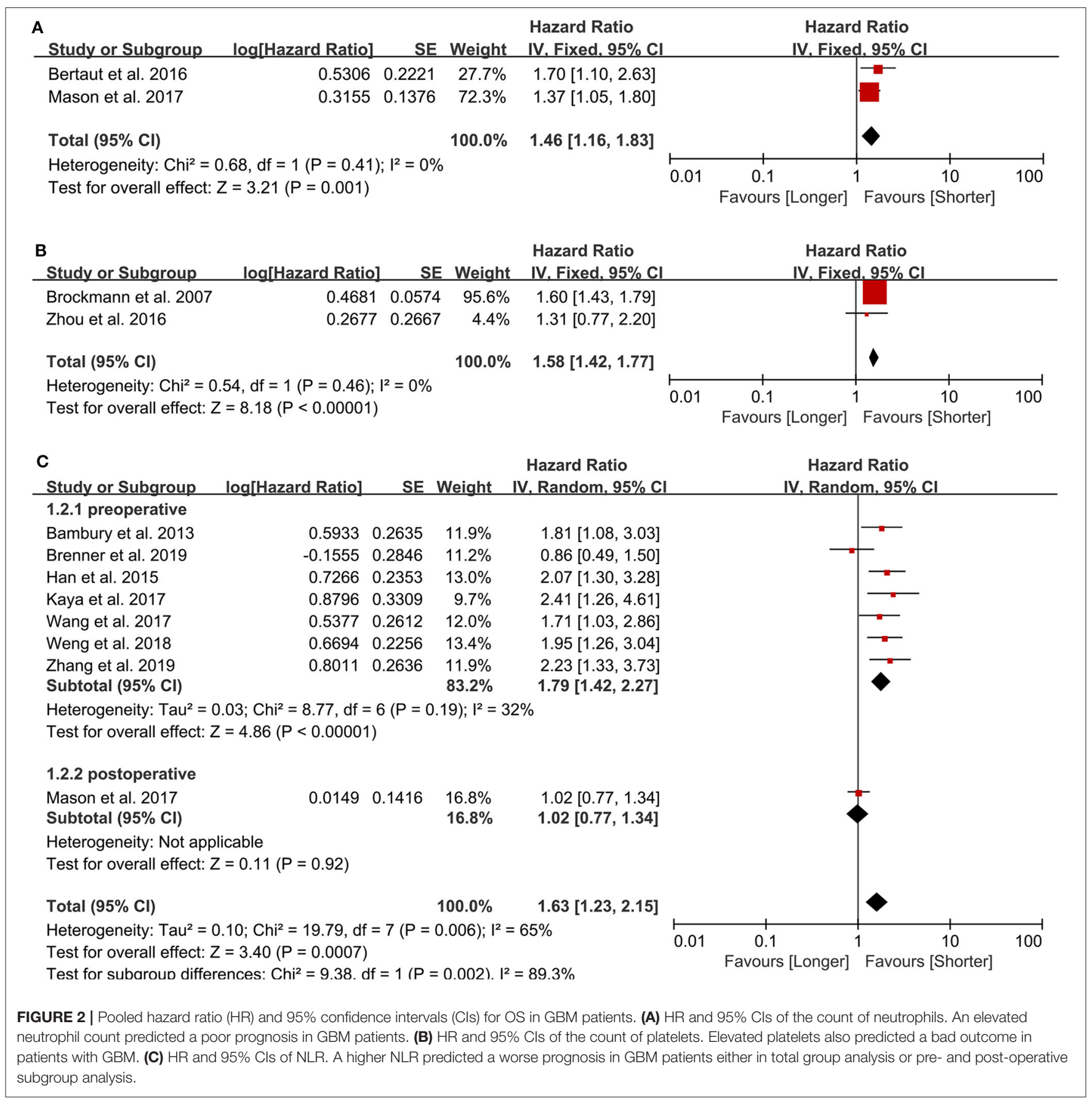

\section{DISCUSSION}

The interplay between inflammation and cancer has been widely investigated in the past few decades. Tumor progression is greatly influenced by inflammatory cells, and inflammation may drive the secretion of some crucial pro-tumorigenic signals, including growth factors and pro-angiogenic factors (5). Inflammation may also accelerate the development of glioma $(38,39)$. Neutrophils, lymphocytes, and platelets and the NLR and PLR are all markers of the systemic inflammatory response. Previous studies have reported the potential prognostic value of inflammatory markers in various tumors. Importantly, peripheral inflammatory markers can be easily measured during routine blood examination after hospitalization. To our best knowledge, this is the first meta-analysis considering all the relevant peripheral inflammation indices based on peripheral blood cells to explore their use as prognostic markers in GBM patients.

In our study, the NLR and the absolute neutrophil count independently predicted the prognosis of GBM patients, 


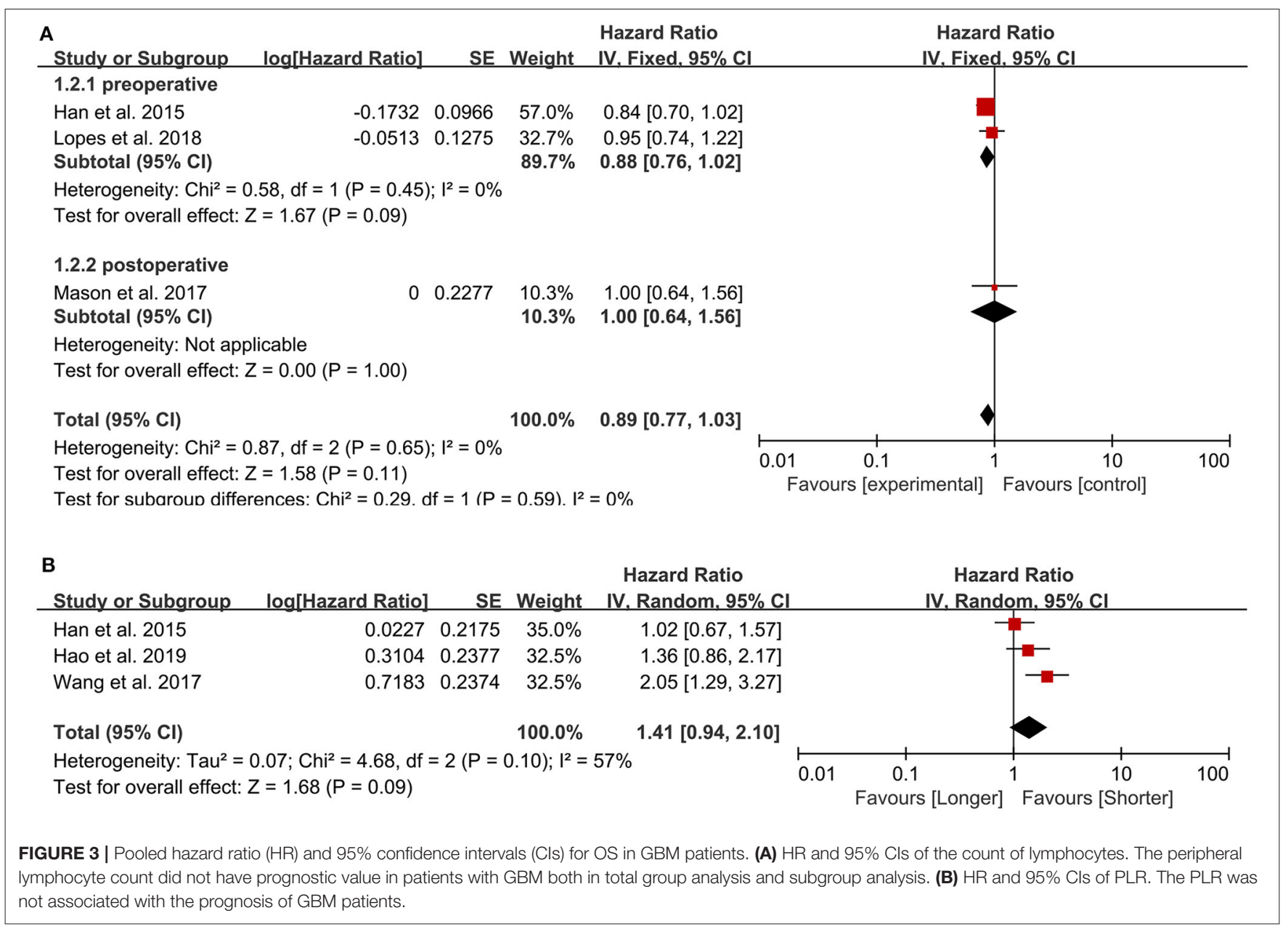

showing their possible values as prognostic biomarkers in GBM. The peripheral blood neutrophil count is frequently high in cancer patients $(31,32,40)$. Neutrophils may promote tumor progression by secreting important cytokines, such as VEGF (41), IL-6 (42), IL-8 (43), elastases (44), and matrix metalloproteinases (45). The extent of neutrophil infiltration is thought to significantly correlate with tumor grade in glioma, and the number of circulating or infiltrating neutrophils around the tumor may be influenced by factors deriving from glioma (46). Tumor cells may increase the neutrophil count both in the peripheral blood and around the tumor by secreting chemotactic factors, such as G-CSF, VEGF, IL-1 $\beta$, and IL-6 (40, 47, 48). A recent study also reported that the blood neutrophil count may predict the efficacy of bevacizumab in GBM (12). An increased neutrophil count is also thought to inhibit lymphocyte survival and suppress the normal cytotoxic function by regulating lymphocyte function via releasing reactive oxygen species and arginase (40). The two studies included in this meta-analysis that analyzed the neutrophil count analyzed it as a categorical variable and used postoperative blood samples. As a result, most patients have likely already been exposed to corticosteroids, which affects both the neutrophil and lymphocyte counts, and the stress from surgery itself may also have an impact on systemic inflammation (31). However, the lymphocyte count was not associated with the prognosis of GBM patients in our study. Additionally, the two studies did not state at which time point after surgery they collected their blood samples, and the cutoff values were different. Interestingly, the decrease rate in the neutrophil count has also been reported to correlate with survival in GBM patients. A decrease rate of $40 \%$ or higher markedly predicts good prognosis of $I D H$-wild type GBM patients in the concomitant TMZ phase (49). Therefore, caution should be applied to this finding, and more studies are needed to verify it.

The NLR, by comprising the neutrophil and lymphocyte counts, is regarded as an index that reflects the balance of the inflammation and immune responses. A meta-analysis performed by Templeton et al. (50) indicated that a higher NLR predicted a poorer OS in many solid tumors. In this study, five studies used an NLR of 4 as the cut-off value, and the pooled result was consistent with previous findings. One study by Kaya et al. used 5 as the cut-off value and found that the NLR was correlated with survival (33), whereas another study by Mason et al. used 7.5 as the cut-off value and found that the NLR was not correlated with survival (11), although they used postoperative samples. Notably, a recent study by Brenner et al. (35) come to the result that the pretreatment NLR did not have the prognostic 


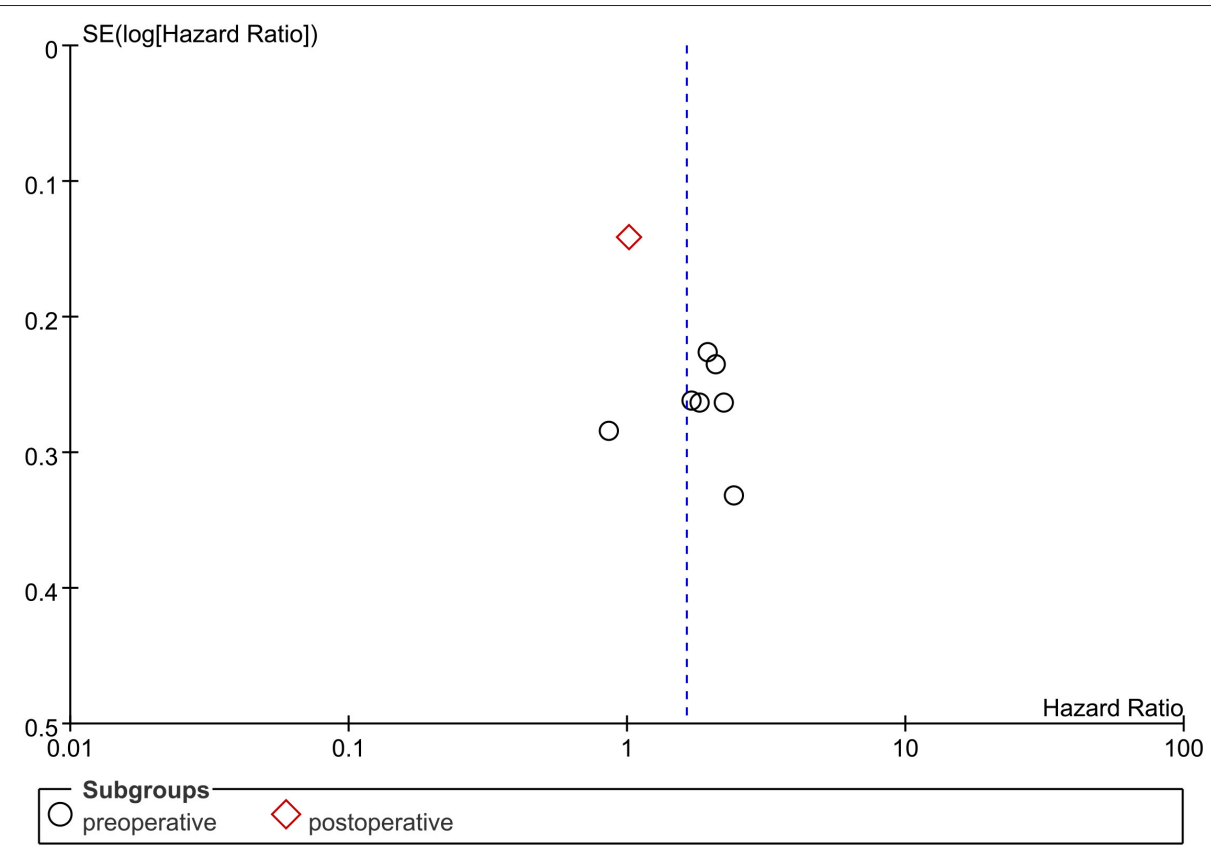

FIGURE 4 | Funnel plot of publication bias of NLR in GBM patients. No significant publication bias was found in the present meta-analysis.

value in glioblastoma patients treated with combined modality surgery, radiation, and temozolomide, indicating the prognostic significance of the NLR in some designate cohort remains unclear. The NLR was also found to positively correlate with the expression of Ki-67 (34) and the glioma grade (51-53). A more exact conclusion from our study may be that a preoperative NLR >4 may predict worse outcome in GBM patients, and the postoperative neutrophil count may be a marker for the prognosis of GBM patients. Nevertheless, we recommend that future studies use preoperative blood samples in order to avoid the potential factors mentioned above. The exact mechanisms by which the NLR influences the prognosis of GBM patients remains unknown.

Another marker of systemic inflammation is the PLR (54), which has also been studied as a prognostic indicator in many cancers, including non-small cell lung cancer (55) and renal cell carcinoma (22). The underlying mechanism of the PLR in GBM is still confusing. An elevated platelet level may boost tumor growth, angiogenesis, and dissemination (56) by secreting crucial factors, such as VEGF (57-59). To our knowledge, this study is the first to evaluate the important roles of the platelet count in glioma cohorts. According to our results, an elevated preoperative platelet count predicts a poorer prognosis in GBM patients. However, the two studies used different cut-off values, which may influence the clinical utility of this finding. More studies are needed to further explore the prognostic value of the preoperative platelet count. However, in this study, the PLR showed no prognostic value in GBM patients, and the heterogeneity was significant $\left(I^{2}=57 \%\right)$. Therefore, caution should be taken in applying this result.
In this study, assessing the neutrophils, lymphocytes, platelets, NLR, and PLR as either categorical or continuous variables may have had an effect on the results. Lopes et al. (13) evaluated these factors as continuous variables and came to the conclusion that none of these markers had prognostic value in GBM patients. Because the other included studies evaluated these markers as categorical variables, this study was excluded from the quantitative synthesis in the analysis of neutrophils, platelets, the NLR, and the PLR. Unlike the other three studies assessing the lymphocyte count, Mendez et al. (14) treated the lymphocyte count as a dichotomous variable, and therefore it was excluded from the quantitative synthesis. We recommend that future studies take notice of the influence of variable types involving different statistical methods on results.

There are also some limitations in our meta-analysis. First, nearly all of the incorporated studies were retrospective, which inevitably causes some bias due to the nature of retrospective analysis. Second, there were only a small number of studies of some markers; therefore, the results should be applied with caution. Moreover, the characteristics among studies varied considerably, including the patients with different race or from different regions, which may be an important source of heterogeneity. More optimally designed studies are needed to validate our results. Although there has been a meta-analysis evaluating the prognostic significance of the NLR in glioma patients, our study focused on the cohort of glioblastoma and included more systemic inflammatory indicators and more independent studies on the NLR in glioblastoma patients, and is therefore valuable, despite these limitations. 


\section{CONCLUSIONS}

In conclusion, the NLR and the neutrophil and platelet counts may be valuable and convenient peripheral inflammatory markers to evaluate the prognosis of GBM patients. However, more prospective and large-scale studies are needed to verify our results. The complex relationship between the GBM microenvironment and systemic inflammatory responses should be profoundly investigated in order to clarify the mechanisms of these inflammatory markers in GBM.

\section{DATA AVAILABILITY STATEMENT}

The raw data supporting the conclusions of this article will be made available by the authors, without undue reservation.

\section{ETHICS STATEMENT}

The studies involving human participants were reviewed and approved by Ethics Committee of Zhongnan Hospital of Wuhan University. The patients/participants provided their written informed consent to participate in this study.

\section{AUTHOR CONTRIBUTIONS}

CY: took responsibility for the integrity of the data and the accuracy of the data analysis. CY and H-BW: drafted the

\section{REFERENCES}

1. Stupp R, Mason WP, van den Bent MJ, Weller M, Fisher B, Taphoorn $\mathrm{MJ}$, et al. Radiotherapy plus concomitant and adjuvant temozolomide for glioblastoma. N Engl J Med. (2005) 352:987-96. doi: 10.1056/NEJMoa0 43330

2. Diamandis $\mathrm{P}$, Aldape KD. Insights from molecular profiling of adult glioma. $J$ Clin Oncol. (2017) 35:2386-93. doi: 10.1200/JCO.2017.73.9516

3. Reifenberger G, Wirsching HG, Knobbe-Thomsen CB, Weller M. Advances in the molecular genetics of gliomas - implications for classification and therapy. Nat Rev Clin Oncol. (2017) 14:434-52. doi: 10.1038/nrclinonc.20 16.204

4. Louis DN, Perry A, Reifenberger G, von Deimling A, Figarella-Branger D, Cavenee WK, et al. The 2016 World Health Organization classification of tumors of the central nervous system: a summary. Acta Neuropathol. (2016) 131:803-20. doi: 10.1007/s00401-016-1545-1

5. Hanahan D, Weinberg RA. Hallmarks of cancer: the next generation. Cell. (2011) 144:646-74. doi: 10.1016/j.cell.2011.02.013

6. Joyce JA, Pollard JW. Microenvironmental regulation of metastasis. Nat Rev Cancer. (2009) 9:239-52. doi: 10.1038/nrc2618

7. Massara M, Persico P, Bonavita O, Mollica Poeta V, Locati M, Simonelli M, et al. Neutrophils in gliomas. Front Immunol. (2017) 8:1349. doi: 10.3389/fimmu.2017.01349

8. Donskov F. Immunomonitoring and prognostic relevance of neutrophils in clinical trials. Semin Cancer Biol. (2013) 23:2007. doi: 10.1016/j.semcancer.2013.02.001

9. Kaneko M, Nozawa H, Sasaki K, Hongo K, Hiyoshi M, Tada N, et al. Elevated neutrophil to lymphocyte ratio predicts poor prognosis in advanced colorectal cancer patients receiving oxaliplatin-based chemotherapy. Oncology. (2012) 82:261-8. doi: 10.1159/000337228

10. Zhang YJ, Peng ZW, Chen MX, Liu FR, Huang JT, Xu L, et al. Elevated neutrophil to lymphocyte ratio might predict poor prognosis for colorectal liver metastasis after percutaneous radiofrequency ablation. manuscript. CY and Y-HZ: statistical analysis. CY, H-BW, Y-HZ, and $\mathrm{W}-\mathrm{HH}$ : critical revision of the manuscript for important intellectual content. Z-FW and Z-QL: supervision. All authors: concept, design, analysis and interpretation of data.

\section{FUNDING}

This research was supported by grants from National Natural Science Foundation of China (No. 81573459), National Health Commission of China (2018ZX-07S-011), Medical Science Advancement Program of Wuhan University (No. TFJC2018003), and Translational Medicine and Interdisciplinary Research Joint Fund of Zhongnan Hospital of Wuhan University (Nos. ZLYNXM202011, ZNLH201901).

\section{ACKNOWLEDGMENTS}

We would like to thank the Taylor \& Francis Group for their English-language editing service.

\section{SUPPLEMENTARY MATERIAL}

The Supplementary Material for this article can be found online at: https://www.frontiersin.org/articles/10.3389/fneur. 2020.580101/full\#supplementary-material

Int J Hyperthermia. (2012) 28:132-40. doi: 10.3109/02656736.2011.6 54374

11. Mason M, Maurice C, McNamara MG, Tieu MT, Lwin Z, Millar BA, et al. Neutrophil-lymphocyte ratio dynamics during concurrent chemoradiotherapy for glioblastoma is an independent predictor for overall survival. J Neuro-Oncol. (2017) 132:463-71. doi: 10.1007/s11060-0172395-y

12. Bertaut A, Truntzer C, Madkouri R, Kaderbhai CG, Derangere V, Vincent $\mathrm{J}$, et al. Blood baseline neutrophil count predicts bevacizumab efficacy in glioblastoma. Oncotarget. (2016) 7:70948-58. doi: 10.18632/oncotarget.10898

13. Lopes M, Carvalho B, Vaz R, Linhares P. Influence of neutrophil-lymphocyte ratio in prognosis of glioblastoma multiforme. J Neuro-Oncol. (2018) 136:17380. doi: 10.1007/s11060-017-2641-3

14. Mendez JS, Govindan A, Leong J, Gao F, Huang J, Campian JL. Association between treatment-related lymphopenia and overall survival in elderly patients with newly diagnosed glioblastoma. J Neuro-Oncol. (2016) 127:32935. doi: 10.1007/s11060-015-2037-1

15. Buergy D, Wenz F, Groden C, Brockmann MA. Tumor-platelet interaction in solid tumors. Int $J$ Cancer. (2012) 130:2747-60. doi: 10.1002/ij c. 27441

16. Zhou XW, Dong $\mathrm{H}$, Yang $\mathrm{Y}$, Luo JW, Wang $\mathrm{X}$, Liu $\mathrm{YH}$, et al. Significance of the prognostic nutritional index in patients with glioblastoma: a retrospective study. Clin Neurol Neurosurg. (2016) 151:86-91. doi: 10.1016/j.clineuro.2016.10.014

17. Shimada H, Takiguchi N, Kainuma O, Soda H, Ikeda A, Cho A, et al. High preoperative neutrophil-lymphocyte ratio predicts poor survival in patients with gastric cancer. Gastric Cancer. (2010) 13:170-6. doi: 10.1007/s10120-010-0554-3

18. Chen J, Deng Q, Pan Y, He B, Ying H, Sun H, et al. Prognostic value of neutrophil-to-lymphocyte ratio in breast cancer. FEBS Open Biol. (2015) 5:502-7. doi: 10.1016/j.fob.2015.05.003

19. Xue TC, Zhang L, Xie XY, Ge NL, Li LX, Zhang BH, et al. Prognostic significance of the neutrophil-to-lymphocyte ratio 
in primary liver cancer: a meta-analysis. PLoS ONE. (2014) 9:e96072. doi: 10.1371/journal.pone.0096072

20. Sato H, Tsubosa Y, Kawano T. Correlation between the pretherapeutic neutrophil to lymphocyte ratio and the pathologic response to neoadjuvant chemotherapy in patients with advanced esophageal cancer. World J Surg. (2012) 36:617-22. doi: 10.1007/s00268-011-1411-1

21. Templeton AJ, Pezaro C, Omlin A, McNamara MG, Leibowitz-Amit R, VeraBadillo FE, et al. Simple prognostic score for metastatic castration-resistant prostate cancer with incorporation of neutrophil-to-lymphocyte ratio. Cancer. (2014) 120:3346-52. doi: 10.1002/cncr.28890

22. Hu H, Yao X, Xie X, Wu X, Zheng C, Xia W, et al. Prognostic value of preoperative NLR, dNLR, PLR and CRP in surgical renal cell carcinoma patients. World J Urol. (2017) 35:261-70. doi: 10.1007/s00345-0161864-9

23. Wick W, Meisner C, Hentschel B, Platten M, Schilling A, Wiestler B, et al. Prognostic or predictive value of MGMT promoter methylation in gliomas depends on IDH1 mutation. Neurology. (2013) 81:151522. doi: 10.1212/WNL.0b013e3182a95680

24. Lei YY, Li YT, Hu QL, Wang J Sui AX. Prognostic impact of neutrophil-to-lymphocyte ratio in gliomas: a systematic review and metaanalysis. World J Surg Oncol. (2019) 17:152. doi: 10.1186/s12957-0191686-5

25. Wang DP, Kang K, Lin Q Hai J. Prognostic significance of preoperative systemic cellular inflammatory markers in gliomas: a systematic review and meta-analysis. Clin Transl Sci. (2020) 13:179-88. doi: 10.1111/ct s. 12700

26. Moher D, Liberati A, Tetzlaff J, Altman DG, Group P. Preferred reporting items for systematic reviews and meta-analyses: the PRISMA statement. Ann Intern Med. (2009) 151:264-9, W64. doi: 10.7326/0003-4819-151-4-200908180-00135

27. Higgins JP, Thompson SG, Deeks JJ, Altman DG. Measuring inconsistency in meta-analyses. BMJ. (2003) 327:557-60. doi: $10.1136 / \mathrm{bmj} .327 .7$ 414.557

28. Han S, Huang Y, Li Z, Hou H, Wu A. The prognostic role of preoperative serum albumin levels in glioblastoma patients. BMC Cancer. (2015) 15:108. doi: 10.1186/s12885-015-1125-0

29. Brockmann MA, Giese A, Mueller K, Kaba FJ, Lohr F, Weiss C, et al. Preoperative thrombocytosis predicts poor survival in patients with glioblastoma. Neuro-Oncol. (2007) 9:335-42. doi: 10.1215/152285172007-013

30. Wang PF, Song HW, Cai HQ, Kong LW, Yao K, Jiang T, et al. Preoperative inflammation markers and IDH mutation status predict glioblastoma patient survival. Oncotarget. (2017) 8:50117-23. doi: 10.18632/oncotarge t. 15235

31. Han S, Liu Y, Li Q, Li Z, Hou H, Wu A. Pre-treatment neutrophil-tolymphocyte ratio is associated with neutrophil and T-cell infiltration and predicts clinical outcome in patients with glioblastoma. BMC Cancer. (2015) 15:617. doi: 10.1186/s12885-015-1629-7

32. Bambury RM, Teo MY, Power DG, Yusuf A, Murray S, Battley JE, et al. The association of pre-treatment neutrophil to lymphocyte ratio with overall survival in patients with glioblastoma multiforme. J Neuro-Oncol. (2013) 114:149-54. doi: 10.1007/s11060-013-1164-9

33. Kaya V, Yildirim M, Yazici G, Yalcin AY, Orhan N, Guzel A. Prognostic significance of indicators of systemic inflammatory responses in glioblastoma patients. Asian Pacific J Cancer Prevent. (2017) 18:3287-91. doi: 10.1093/annonc/mdx366.026

34. Weng W, Chen X, Gong S, Guo L, Zhang X. Preoperative neutrophillymphocyte ratio correlated with glioma grading and glioblastoma survival. Neurol Res. (2018) 40:917-22. doi: 10.1080/01616412.2018.14 97271

35. Brenner A, Friger M, Geffen David B, Kaisman-Elbaz T, Lavrenkov K. The prognostic value of the pretreatment neutrophil/lymphocyte ratio in patients with glioblastoma multiforme brain tumors: a retrospective cohort study of patients treated with combined modality surgery, radiation therapy, and temozolomide chemotherapy. Oncology. (2019) 97:25563. doi: $10.1159 / 000500926$

36. Zhang ZY, Zhan YB, Zhang FJ, Yu B, Ji YC, Zhou JQ, et al. Prognostic value of preoperative hematological markers combined with molecular pathology in patients with diffuse gliomas. AGING. (2019) 11:625272. doi: 10.18632/aging. 102186

37. Hao YF, Li XL, Chen HC, Huo HZ, Liu ZB, Tian F, et al. A cumulative score based on preoperative neutrophil-lymphocyte ratio and fibrinogen in predicting overall survival of patients with glioblastoma multiforme. World Neurosurg. (2019) 128:e427-33. doi: 10.1016/j.wneu.201 9.04.169

38. Yeung YT, McDonald KL, Grewal T, Munoz L. Interleukins in glioblastoma pathophysiology: implications for therapy. Br J Pharmacol. (2013) 168:591606. doi: 10.1111/bph.12008

39. Linos E, Raine T, Alonso A, Michaud D. Atopy and risk of brain tumors: a meta-analysis. J Natl Cancer Instit. (2007) 99:1544-50. doi: 10.1093/jnci/djm170

40. Gregory AD, Houghton AM. Tumor-associated neutrophils: new targets for cancer therapy. Cancer Res. (2011) 71:24116. doi: 10.1158/0008-5472.CAN-10-2583

41. McCourt M, Wang JH, Sookhai S, Redmond HP. Proinflammatory mediators stimulate neutrophil-directed angiogenesis. Arch Surg. (1999) 134:132531. doi: 10.1001/archsurg.134.12.1325

42. Jabłońska E, Kiluk M, Markiewicz W, Piotrowski L, Grabowska Z, Jabłoński J. TNF- $\alpha$, IL-6 and their soluble receptor serum levels and secretion by neutrophils in cancer patients. Arch Immunol Therap Exp. (2001) 49:63-9.

43. Schaider H, Oka M, Bogenrieder T, Nesbit M, Satyamoorthy K, Berking C, et al. Differential response of primary and metastatic melanomas to neutrophils attracted by IL-8. Int J Cancer. (2003) 103:335-43. doi: 10.1002/ij c. 10775

44. Scapini P, Nesi L, Morini M, Tanghetti E, Belleri M, Noonan D, et al. Generation of biologically active angiostatin kringle 1-3 by activated human neutrophils. J Immunol. (2002) 168:5798804. doi: 10.4049/jimmunol.168.11.5798

45. Shamamian P, Schwartz JD, Pocock BJ, Monea S, Whiting D, Marcus SG, et al. Activation of progelatinase A (MMP-2) by neutrophil elastase, cathepsin G, and proteinase-3: a role for inflammatory cells in tumor invasion and angiogenesis. J Cell Physiol. (2001) 189:197-206. doi: 10.1002/jc p.10014

46. Fossati G, Ricevuti G, Edwards SW, Walker C, Dalton A, Rossi ML. Neutrophil infiltration into human gliomas. Acta Neuropathol. (1999) 98:34954. doi: $10.1007 / \mathrm{s} 004010051093$

47. Annalisa DP, Paola A, Giuseppe S, Ruggiero F, Massimiliano M, Alberto M. Molecular pathways in cancer-related inflammation. Biochem Med. (2011) 21:264-75. doi: 10.11613/BM.2011.036

48. Lechner MG, Liebertz DJ, Epstein AL. Characterization of cytokine-induced myeloid-derived suppressor cells from normal human peripheral blood mononuclear cells. J Immunol. (2010) 185:2273-84. doi: 10.4049/jimmunol.1000901

49. Saito T, Sugiyama K, Hama S, Yamasaki F, Takayasu T, Nosaka R, et al. Prognostic importance of temozolomide-induced neutropenia in glioblastoma, IDH-wildtype patients. Neurosurg Rev. (2018) 41:621-8. doi: 10.1007/s10143-017-0903-3

50. Templeton AJ, McNamara MG, Seruga B, Vera-Badillo FE, Aneja P, Ocana A, et al. Prognostic role of neutrophil-to-lymphocyte ratio in solid tumors: a systematic review and meta-analysis. J Natl Cancer Instit. (2014) 106:dju124. doi: 10.1093/jnci/dju124

51. Wang JL, Xiao WJ, Chen WY, Hu YH. Prognostic significance of preoperative neutrophil-to-lymphocyte ratio and platelet-to-lymphocyte ratio in patients with glioma. EXCLI J. (2018) 17:505-12. doi: 10.17179/excli2017-978

52. Zadora P, Dabrowski W, Czarko K, Smolen A, Kotlinska-Hasiec E, Wiorkowski K, et al. Preoperative neutrophil-lymphocyte count ratio helps predict the grade of glial tumor - a pilot study. Neurol Neurochir Pol. (2015) 49:41-4. doi: 10.1016/j.pjnns.2014.12.006

53. Weng Y, Zhang X, Han J, Ouyang L, Liang M, Shi Z, et al. Do selected blood inflammatory markers combined with radiological features predict proliferation index in glioma patients? World Neurosurg. (2018) 118:e13746. doi: 10.1016/j.wneu.2018.06.142

54. McMillan DC. An inflammation-based prognostic score and its role in the nutrition-based management of patients with cancer. Proc Nutr Soc. (2008) 67:257-62. doi: 10.1017/S0029665108007131 
55. Kang KH, Efird JT, Sharma N, Yang M, Dowlati A, Linden P, et al. Prognostic potential of neutrophil-to- lymphocyte ratio and lymphocyte nadir in stage III non-small-cell lung cancer. Future Oncol. (2017) 13:140514. doi: $10.2217 /$ fon-2017-0045

56. Menter DG, Tucker SC, Kopetz S, Sood AK, Crissman JD, Honn KV. Platelets and cancer: a casual or causal relationship: revisited. Cancer Metastasis Rev. (2014) 33:231-69. doi: 10.1007/s10555-0149498-0

57. Vito CD, Navone SE, Marfia G, Hadi AL, Mancuso ME, Pecci A, et al. Platelets from glioblastoma patients promote angiogenesis of tumor endothelial cells and exhibit increased VEGF content and release. Platelets. (2017) 28:58594. doi: 10.1080/09537104.2016.1247208

58. Westermark B, Heldin $\mathrm{CH}$, Nistér M. Platelet-derived growth factor in human glioma. GLIA. (1995) 15:257-63. doi: 10.1002/glia.440 150307
59. Wojtukiewicz MZ, Sierko E, Hempel D, Tucker SC, Honn KV. Platelets and cancer angiogenesis nexus. Cancer Metastasis Rev. (2017) 36:24962. doi: $10.1007 /$ s10555-017-9673-1

Conflict of Interest: The authors declare that the research was conducted in the absence of any commercial or financial relationships that could be construed as a potential conflict of interest.

Copyright (c) 2020 Yang, Wen, Zhao, Huang, Wang and Li. This is an open-access article distributed under the terms of the Creative Commons Attribution License (CC $B Y)$. The use, distribution or reproduction in other forums is permitted, provided the original author(s) and the copyright owner(s) are credited and that the original publication in this journal is cited, in accordance with accepted academic practice. No use, distribution or reproduction is permitted which does not comply with these terms. 